\title{
Highly Luminescent CdTe/CdSe Colloidal Heteronanocrystals with Temperature-Dependent Emission Color
}

\author{
Patrick T. K. Chin, ${ }^{\dagger}$ Celso de Mello Donegá,,,$\|$ Svetlana S. van Bavel, ${ }^{\ddagger}$ \\ Stefan C. J. Meskers, ${ }^{\dagger}$ Nico A. J. M. Sommerdijk, ${ }^{\S}$ and René A. J. Janssen ${ }^{*} \dagger$ \\ Contribution from the Molecular Materials and Nanosystems, Materials and Interface Chemistry, \\ and Soft Matter Cryo TEM Research Unit, Eindhoven University of Technology, P.O. Box 513, \\ 5600 MB Eindhoven, The Netherlands, and Condensed Matter and Interfaces, Debye Institute, \\ Utrecht University, P.O. Box 80000, 3508 TA Utrecht, The Netherlands
}

Received May 26, 2007; E-mail: r.a.j.janssen@tue.nl; c.demellodonega@phys.uu.nl

\begin{abstract}
In this work we present the preparation of highly luminescent anisotropic $\mathrm{CdTe} / \mathrm{CdSe}$ colloidal heteronanocrystals. The reaction conditions used (low temperature, slow precursor addition, and surfactant composition) resulted in a tunable shape from prolate to branched $\mathrm{CdTe} / \mathrm{CdSe}$ nanocrystals. Upon CdSe shell growth the heteronanocrystals show a gradual evolution from type-I to type-II optical behavior. These heteronanocrystals show a remarkably high photoluminescence quantum yield (up to $82 \%$ ) and negligible thermally induced quenching up to temperatures as high as $373 \mathrm{~K}$.
\end{abstract}

\section{Introduction}

The remarkable size and shape-dependent properties of semiconductor nanostructures ${ }^{1}$ have attracted increasing interest over the past decades, and as a result, an excellent degree of control over the composition, size, shape, and surface of semiconductor nanocrystals has been achieved in recent years by using colloidal chemistry routes. ${ }^{2}$ Semiconductor heteronanostructures, such as core/shell quantum dots (QDs), can show different behavior regarding charge carrier localization after photoexcitation (type-I or type-II), depending on the band offset alignments between the core and the shell materials. ${ }^{3}$ In type-I QDs both carriers are primarily confined in the same part of the heterostructure, while in type-II QDs the band offsets are such that electrons and holes are spatially separated, leading to the formation of an indirect exciton. The radiative recombination of the indirect exciton will result in emission at lower energies than those of both the core and the shell optical gaps, thus allowing access to wavelengths that would otherwise not be available with a single material or type-I QDs. Since the position of the energy levels of QDs is strongly size-dependent, ${ }^{1,2}$ the relative energy offsets can be tuned by controlling the core diameter and the shell thickness. This offers the possibility of directly controlling the degree of charge carrier localization from

† Molecular Materials and Nanosystems, Eindhoven University of Technology.

$\doteqdot$ Materials and Interface Chemistry, Eindhoven University of Technology.

$\S$ Soft Matter Cryo TEM Research Unit, Eindhoven University of Technology.

"Utrecht University.

(1) Alivisatos, A. P. J. Phys. Chem. 1996, 100, 13226-13239.

(2) de Mello Donegá, C.; Liljeroth, P.; Vanmaekelbergh, D. Small 2005, 1, $1152-1162$

(3) Hatami, F.; Grundmann, M.; Ledentsov, N. N.; Heinrichsdorff, F.; Heitz, R.; Böhrer, J.; Bimberg, D.; Ruvimov, S. S.; Werner, P.; Ustinov, V. M.; Kopev, P. S.; Alferov, Zh. I. Phys. Rev. B 1998, 57, 4635-4641.
type-I to type-II, ${ }^{4}$ and consequently the emission wavelength, exciton radiative lifetimes and the exciton confinement energies, with important consequences for a number of potential applications such as photovoltaic devices, photocatalysts, lasers, and LEDs.

The potential advantages of colloidal type-II QDs have triggered an increasing interest in the past few years, ${ }^{4-17}$ and several semiconductor combinations have been investigated as prospective type-II QDs, based on the band offsets of the bulk materials (viz., $\mathrm{ZnSe}-\mathrm{CdSe},{ }^{4-5} \mathrm{CdTe}-\mathrm{CdSe},{ }^{6-12} \mathrm{ZnTe}-$ $\left.\mathrm{CdSe}^{6,13-15}\right)$. These pioneering studies ${ }^{4-17}$ have provided a wealth of new insights into both the chemistry and the physics of colloidal type-II QDs, but there are still several challenging issues to be addressed. For example, the photoluminescence quantum yields (QYs) reported for colloidal type-II QDs are

(4) Balet, L. P.; Ivanov, S. A.; Piryatinski, A.; Achermann, M.; Klimov, V. I. Nano Lett. 2004, 4, 1485-1488.

(5) Nanda, J.; Ivanov, S. A.; Htoon, H.; Bezel, I.; Piryatinski, A.; Tretiak, S.; Klimov, V. I. J. Appl. Phys. 2006, 99, 034309/1-7.

(6) Kim, S.; Fisher, B.; Eisler, H-J.; Bawendi, M. J. Am. Chem. Soc. 2003 $125,11466-11467$

(7) Li, J. J.; Tsay, J. M.; Michalet, X.; Weiss, S. Chem. Phys. 2005, 318, 8290

(8) Chou, P. T.; Chen, C. Y.; Cheng, C. T.; Pu, S. C.; Wu, K. C.; Cheng, Y. M.; Lai, C. W.; Chou, Y. H.; Chiu, H. T. Chem. Phys. Chem. 2006, 7 $222-228$.

(9) Oron, D.; Kazes, M.; Banin, U. Phys. Rev. B 2007, 75, 035330/1-7.

(10) Milliron, D. J.; Hughes, S. M.; Cui, Y.; Manna, L.; Li, J.; Wang, L. W.; Alivisatos, A. P. Nature 2004, 430, 190-195.

(11) Shieh, F.; Saunders, A. E.; Korgel, B. A. J. Phys. Chem. B 2005, 109 8538-8542.

(12) Halpert, J. E.; Porter, V. J.; Zimmer, J. P.; Bawendi, M. G. J. Am. Chem. Soc. 2006, 128, 12590-12591.

(13) Chen, C. Y.; Cheng, C. T.; Yu, J. K.; Pu, S. C.; Cheng, Y. M.; Chou, P. T.; Chou, Y. H.; Chiu, H. T. J. Phys. Chem. B 2004, 108, 10687-10691.

(14) Chen, C. Y.; Cheng, C. T.; Lai, C. W.; Hu, Y. H.; Chou, P. T.; Chou, Y. H.; Chiu, H. T. Small 2005, 1, 1215-1220.

(15) Xie, R.; Zhong, X.; Basché, T. Adv. Mater. 2005, 17, 2741-2745.

(16) Yu, K.; Zaman, B.; Romanova, S.; Wang, D.; Ripmeester, J. A. Small 2005 1, 332-338.

(17) Ivanov, S. A.; Piryatinski, A.; Nanda, J.; Tretiak, S.; Zavadil, K. R.; Wallace, W. O.; Werder, D.; Klimov, V. I. J. Am. Chem. Soc. 2007, 129, 1170811719. 
typically very low $\left(0-10 \%{ }^{6,8,10-16}\right)$. High QYs $(30-40 \%)$ have been reported for thin shell heteronanocrystals of both $\mathrm{CdTe} /$ $\mathrm{CdSe}$ and $\mathrm{ZnTe} / \mathrm{CdSe} .{ }^{15,16}$ However, these thin shell heteronanocrystals still present a type-I character, as they do not show the featureless subband-gap absorption tail indicative of the spatially indirect transitions expected for type-II QDs. ${ }^{6,10} \mathrm{With}$ increasing shell thickness the long absorption tail appears but the transition from type-I to type-II behavior is accompanied by a strong decrease in QY to below 10\%. These low QYs have been seen as an intrinsic limitation of type-II QDs, ${ }^{6,12}$ since the slower radiative recombination of indirect excitons would facilitate the dominance of nonradiative recombination at defects. However, preparation methodologies that minimize both surface and interfacial defects may substantially improve the photoluminescence QYs of type-II QDs beyond its low current values. Accordingly, a recent report describes highly luminescent $\mathrm{CdS} / \mathrm{ZnSe}$ core/shell type-II nanocrystals with emission QYs up to $50 \%$ that benefit from an intermediate $\mathrm{ZnCdSe}$ alloy layer with a graded composition. ${ }^{17}$ Here we describe the synthesis and properties of highly luminescent $\mathrm{CdTe} / \mathrm{CdSe}$ core/shell nanocrystals (NCs). The synthesis method developed in this work yields highly efficient (PL QY up to $80 \%$ at $300 \mathrm{~K}$ ) anisotropic $\mathrm{CdTe} / \mathrm{CdSe}$ core/shell NCs, with shapes tunable from prolate type-I QDs (aspect ratio: 1.5-2) to branched heteronanocrystals that exhibit type-II character. The high quality of these $\mathrm{CdTe} / \mathrm{CdSe}$ heteronanocrystals is also attested by the negligible contribution of thermally induced luminescence quenching processes in the $293-383 \mathrm{~K}$ temperature range, allowing the temperature dependence of the emission color to be observed. The results reported here clearly demonstrate that type-II heteronanocrystals can have high photoluminescence QYs, despite the longer radiative lifetimes of indirect excitons.

\section{Experimental Section}

2.1. Chemicals. Trioctylphosphine (TOP, 90\%), trioctylphosphine oxide (TOPO, 99\%), dodecylamine (DDA, 98\%), hexadecylamine (HDA, 90\%), and anhydrous solvents (toluene, methanol, chloroform) were all purchased from Aldrich. Selenium powder (99.99\%, 200 mesh) and anhydrous cadmium acetate (CdAc, 99.99+\%) were purchased from Chempur. Tellurium powder $(99.999 \%,<250 \mu \mathrm{m})$ and dimethylcadmium (99.99\%) were purchased from Heraeus and ARC Technologies, respectively. All chemicals were used as received, with the exception of TOPO, HDA, and DDA, which were dried and degassed before use by heating under vacuum, DDA at $373 \mathrm{~K}$ for $6 \mathrm{~h}$, TOPO and HDA at $393 \mathrm{~K}$ for $1.5 \mathrm{~h}$.

2.2. Synthesis and Experimental Details. High-quality greenemitting CdTe QDs (2.6-2.8 $\mathrm{nm}$ average diameter, 5-10\% size dispersion) were prepared in DDA/TOP following a previously reported method $^{18}$ and subsequently purified by filtration (to remove unreacted $\mathrm{Te})$, precipitation with anhydrous methanol, and drying under vacuum. The purified CdTe QDs $(16 \mathrm{mg})$ were dissolved in toluene $(1 \mathrm{~mL})$ and redispersed in a TOPO $(67 \mathrm{wt} \%)-\mathrm{HDA}(33 \mathrm{wt} \%)$ mixture at $393 \mathrm{~K}$. Previous work has shown that this surfactant mixture allows wellcontrolled growth and yields high-quality CdSe QDs. ${ }^{19}$ The CdSe shell growth is carried out at $423 \mathrm{~K}$ by dropwise $(0.05 \mathrm{~mL} / \mathrm{min})$ and alternate addition of $\mathrm{Cd}(0.86 \mathrm{mmol}$ of $\mathrm{CdAc}$ in $3 \mathrm{~mL}$ of TOP $)$ and $\mathrm{Se}(1.26$ mmol of Se in $3 \mathrm{~mL}$ of TOP) precursors, followed by a $30 \mathrm{~min}$ annealing step at $403 \mathrm{~K}$. Aliquots of the growth solution (Table 1) were taken at regular time intervals during the shell growth and quickly

(18) Wuister, S. F.; van Driel, F.; Meijerink, A. Phys. Chem. Chem. Phys. 2003, $5,1253-1258$.

(19) de Mello Donegá, C.; Hickey, S. G.; Wuister, S. F.; Vanmaekelbergh, D.; Meijerink, A. J. Phys. Chem. B 2003, 107, 489-496.
Table 1. CdTe/CdSe Samples Isolated during Shell Growth ${ }^{2}$

\begin{tabular}{lccclc}
\hline & & & & \multicolumn{2}{c}{ CdSe shell thickness $(\mathrm{nm})$} \\
\cline { 3 - 6 } sample & $t(\mathrm{~min})$ & $\lambda_{\max }(\mathrm{nm})$ & QY $(\%)$ & $\begin{array}{l}\text { length } \\
\text { direction }\end{array}$ & $\begin{array}{c}\text { diameter } \\
\text { direction }\end{array}$ \\
\hline core & & 535 & 7 & & \\
$\mathrm{~A}$ & 5 & 539 & 34 & $0.2^{b}$ & \\
$\mathrm{~B}$ & 15 & 585 & 46 & 0.66 & 0.1 \\
$\mathrm{C}$ & 30 & 630 & 82 & 0.92 & 0.2 \\
$\mathrm{D}$ & 45 & 711 & 52 & $1.6^{c}$ & \\
$\mathrm{E}$ & 90 & 789 & 45 & $6-10^{d}$ & $3^{d}$ \\
\hline
\end{tabular}

${ }^{a}$ Reaction time $(t)$, photoluminescence maximum $\left(\lambda_{\max }\right)$, and quantum yield (QY). CdSe thickness along the particle length and diameter directions. ${ }^{b}$ Tips. ${ }^{c}$ Diagonal direction assuming that the CdTe core occupies the center of the pyramids. ${ }^{d}$ Branch length and diameter.

cooled into room-temperature decalin. No postpreparative procedure was employed after sampling, except for the samples to be investigated by transmission electron microscopy, which were purified by precipitation with anhydrous methanol. All optical measurements were carried out on samples with a low optical density $(\leq 0.1$ at the excitation wavelength). Absorption and PL spectra were collected with a PerkinElmer Lambda 900 spectrophotometer and an Edinburgh Instruments FS920 spectrophotometer, respectively. PL quantum yields were determined using fluorescein in $0.1 \mathrm{M} \mathrm{NaOH}^{20}$ and rhodamine 101 in $0.01 \% \mathrm{HCl}$ ethanolic solution ${ }^{21}$ as references, following the method reported in ref 19. Lifetime measurements were carried out using timecorrelated single photon counting (TCSPC). The nanocrystal dispersions were excited at $400 \mathrm{~nm}$ using a picosecond diode laser (PicoQuant PDL 800B) operated at $0.5-2.5 \mathrm{MHz}$. The detector unit consisted of a Peltier-cooled Hamamatsu microchannel plate photomultiplier (R3809U50). TEM and high-resolution TEM were performed on a TECNAI G ${ }^{2}$ 20 transmission electron microscope (FEI Co., The Netherlands) operated at $200 \mathrm{kV}$ or on a Titan Krios transmission electron microscope (FEI Co., The Netherlands) operated at $300 \mathrm{kV}$. The samples for TEM were prepared by dipping a carbon-coated copper (400-mesh) TEM grid into a chloroform solution of nanocrystals. The excess liquid was removed by blotting using filter paper.

\section{Results and Discussion}

3.1. CdTe/CdSe Heteronanocrystal Growth. The alternate addition of low reactivity precursors (such as cadmium oleate or cadmium acetate) prevents homogeneous nucleation, as has been demonstrated by several recent works employing the SILAR (Successive Ion Layer Adsorption and Reaction) approach ${ }^{22}$ to prepare core-shell QDs ${ }^{7,15,22}$ and core-multishell QDs ${ }^{23-26}$ (e.g., $\left.\mathrm{CdSe}-\mathrm{CdS}-(\mathrm{Cd}, \mathrm{Zn}) \mathrm{S}-\mathrm{ZnS},{ }^{23} \mathrm{CdS}-\mathrm{CdSe}-\mathrm{CdS}^{24,26}\right)$. In contrast to the standard SILAR technique, which is based on alternate injections of enough precursors to yield one monolayer of the cation or anion, ${ }^{22-26}$ in the present work the alternate addition of precursors is carried out dropwise, thus making homogeneous nucleation even more unlikely. The low growth temperature further decreases the reactivity of the precursors, ensuring that heterogeneous nucleation and growth of CdSe at the surface of the CdTe nanocrystals prevails, and also prevents growth of the CdTe cores by Ostwald ripening ${ }^{18}$ or alloying

(20) Strickler, S. J.; Berg, R. A. J. Chem. Phys. 1962, 37, 814-822.

(21) Karstens, T.; Kobs, K. J. Phys. Chem. 1980, 84, 1871-1872.

(22) Li, J. J.; Wang, Y. A.; Guo, W.; Keay, J. C.; Mishima, T. D.; Johnson, M. B.; Peng, X. J. Am. Chem. Soc. 2003, 125, 12567-12575.

(23) Xie, R.; Kolb, U.; Li, J.; Basché, T.; Mews, A. J. Am. Chem. Soc. 2005 127, 7480-7488.

(24) Battaglia, D.; Li, J. J.; Wang, Y.; Peng, X. Angew. Chem., Int. Ed. 2003 42, 5035-5039.

(25) Battaglia, D.; Blackman, B.; Peng, X. J. Am. Chem. Soc. 2005, 127, 1088910897.

(26) Schill, A. W.; Gaddis, C. S.; Qian, W.; El-Sayed, M. A.; Cai, Y.; Milam, V. T.; Sandhage, K. Nano Lett. 2006, 6, 1940-1949. 

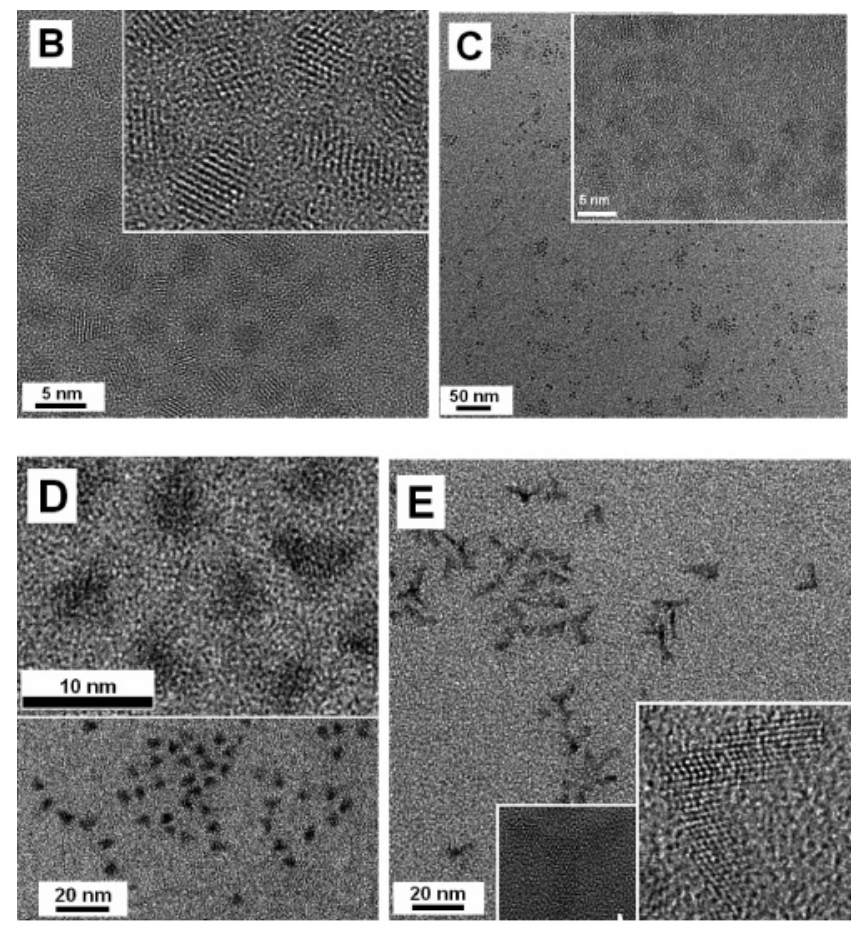

Figure 1. Overview TEM images of $\mathrm{CdTe} / \mathrm{CdSe}$ heteronanocrystal samples B-E (see Table 1) collected at different stages during the CdSe shell growth. Insets show corresponding HRTEM images.

due to interdiffusion. ${ }^{27}$ Therefore, any change in the size (or shape) of the nanocrystals can be ascribed to the shell growth.

As a representative example, Figure 1 shows TEM images of samples collected at different stages during the CdSe shell growth on spherical CdTe cores with a diameter of $2.6 \mathrm{~nm}(5 \%$ size dispersion). It can be seen that the growth is highly anisotropic, initially yielding prolate heteronanocrystals (Figure 1, sample B, diameter: $2.7 \mathrm{~nm}$; length: $3.9 \mathrm{~nm}$, implying the growth of $0.66 \mathrm{~nm} \mathrm{CdSe}$ "tips" in the length direction only), which evolve in trigonal pyramids (Figure 1, sample D, side length: $5.2 \mathrm{~nm}$, implying a $1.6 \mathrm{~nm}$ CdSe shell under the assumption that the CdTe core occupies the center of the pyramids) and eventually undergo branching producing multipods (Figure 1, sample E; mostly bipods, but tripods, rods, matchsticks, and more complex multibranched shapes are also present; branch diameter: $3 \mathrm{~nm}$; branch length: $6-10 \mathrm{~nm}$ ).

The anisotropic growth can be understood considering the set of reaction conditions used in this work (viz., low reactivity precursors, low reaction temperature, slow precursor addition, coordinating solvent with higher affinity for $\mathrm{Cd}$ than for $\mathrm{Se}$ ). Under these conditions the CdSe heteroepitaxial growth is slow and kinetically controlled (i.e., the rate-limiting step is the $\mathrm{CdSe}$ formation at the surface, rather than the diffusion of precursors), and therefore the higher reactivity of the $\{000 \overline{1}\}$ Se terminated polar facets of the CdSe wurtzite structure will favor anisotropic growth along the $c$-direction. ${ }^{28-31}$ The growth evolution toward branched heterostructures, such as the $\mathrm{CdTe} / \mathrm{CdSe}$ bipods and tripods shown in Figure 1 for sample E, is probably due to the

(27) Bailey, R. E.; Nie, S. J. Am. Chem. Soc. 2003, 125, 7100-7106.

(28) Yin, Y.; Alivisatos, A. P. Nature 2005, 437, 664-670.

(29) Peng, X. Adv. Mater. 2003, 15, 459-463.

(30) Rempel, J. Y.; Trout, B. L.; Bawendi, M. G.; Jensen, K. F. J. Phys. Chem. B 2005, 109, 19320-19328.

(31) Talapin, D. V.; Koeppe, R.; Götzinger, S.; Kornowski, A.; Lupton, J. M.; Rogach, A. L.; Benson, O.; Feldmann, J.; Weller, H. Nano Lett. 2003, 3 , $1677-1681$

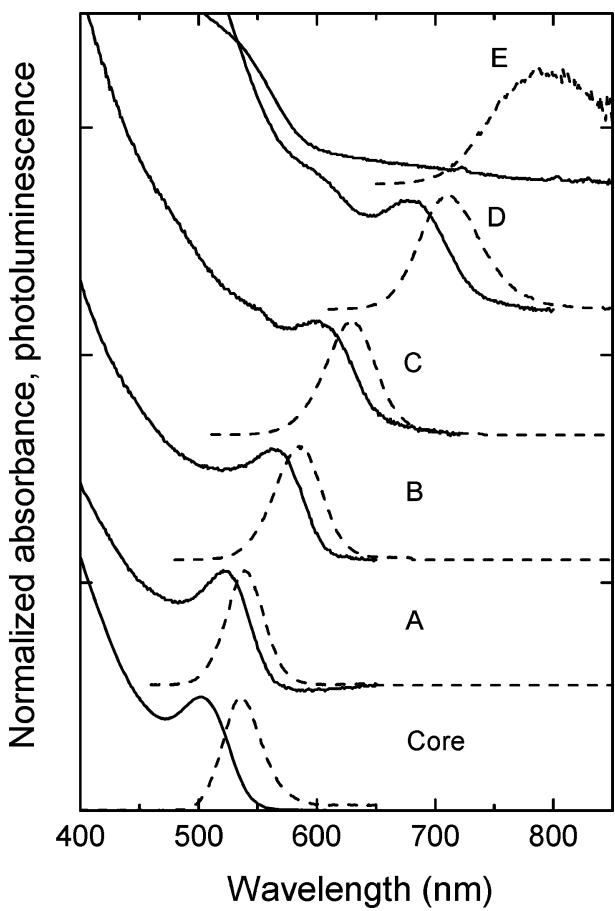

Figure 2. Absorption (solid lines) and photoluminescence (dashed lines) spectra of colloidal CdTe/CdSe heteronanocrystals with a $2.6 \mathrm{~nm} \mathrm{CdTe}$ core and increasing CdSe shell dimension. Labels correspond to Table 1. Spectra are shifted vertically for clarity.

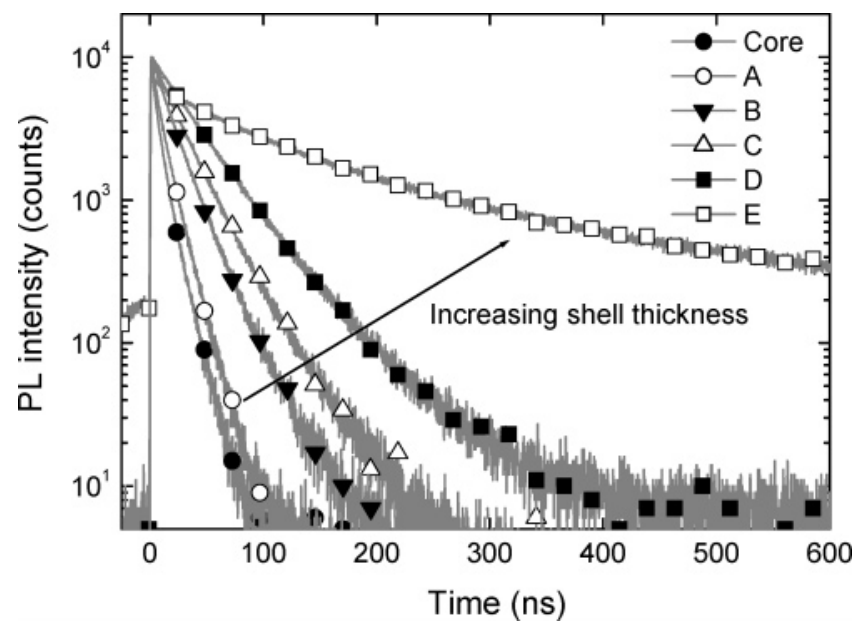

Figure 3. Photoluminescence decay curves of colloidal $\mathrm{CdTe} / \mathrm{CdSe}$ heteronanocrystals with a $2.6 \mathrm{~nm}$ CdTe core and increasing CdSe shell dimensions (labels are explained in Table 1). The decay curve of the CdTe QDs used as cores is also shown (Core).

different crystal structures of the CdTe core (zincblende) and the growing CdSe shell (wurtzite) (see Supporting Information for XRD data). ${ }^{10,28}$

3.2. Optical Properties: Absorption, Photoluminescence, Quantum Yields, and Exciton Lifetimes. Figures 2 and 3 show that the optical properties (viz., absorption, photoluminescence, and exciton lifetimes) of the $\mathrm{CdTe} / \mathrm{CdSe}$ heteronanocrystals change dramatically during the CdSe "shell" growth. The emission wavelength red shifts from $535 \mathrm{~nm}(2.32 \mathrm{eV})$ for the CdTe cores (Figure 2) to $770 \mathrm{~nm}(1.61 \mathrm{eV})$ for the CdTe/CdSe multipods (sample $\mathrm{E}$ in Figures $1-3$ and Table 1). The absorption also red shifts, but as the dimension of the CdSe part of the heteronanocrystal increases, the absorption features are smeared out and the oscillator strengths at the emission 
energies become relatively smaller (Figure 2), while the RT photoluminescence decay times (i.e., the exciton lifetimes) become longer (Figure 3 ). The increase in the exciton lifetime observed for sample CdTe/CdSe B (18 ns, 1-exponential decay; Figure 3) with respect to the CdTe cores (multiexponential decay, average lifetime: $8 \mathrm{~ns}$; Figure 3) is mostly due to the decrease of the nonradiative decay rates, since it is accompanied by a substantial increase in the PL QYs (viz., from $7 \%$ to $46 \%$, Table 1). Moreover, the radiative exciton lifetime for CdTe QDs of similar size (viz., $3.0 \mathrm{~nm}$ ) has been reported to be $18 \mathrm{~ns}^{32}$ Further growth of the CdSe part leads to increasingly longer exciton lifetimes and a change from singleexponential to biexponential decay (viz., Sample C: $24 \mathrm{~ns}$, 1-exp. decay; Sample E: 2-exp. decay, 60 and 220 ns, average lifetime: $100 \mathrm{~ns}$ ). The faster component of the biexponential decay is attributed to $\mathrm{CdTe} / \mathrm{CdSe}$ heteronanocrystals in which nonradiative exciton recombination is also relevant, while the slower component is attributed to purely radiative decay. It is worth noting that the featureless absorption tail observed for the CdTe/CdSe multipods (Figure 2, sample E) is at lower energy than the band edge absorption of both the CdTe core (see Figure 2) and the CdSe branches (565 $\mathrm{nm}$ for CdSe nanorods with $3.2 \mathrm{~nm}$ diameter and $11 \mathrm{~nm}$ length $\left.{ }^{33-34}\right)$. Therefore both the absorption tail and the photoluminescence peak can be unambiguously assigned to an indirect exciton.

These results indicate a progressive reduction of the electronhole wave function overlap and the formation of an indirect exciton as the dimension of the CdSe part of the heteronanocrystal increases (reflected in the progressively longer lifetimes accompanied by a spectral red shift and the development of a featureless subband-gap absorption tail), ultimately leading to a characteristic type-II behavior ${ }^{3-6,9}$ for the CdTe/CdSe multipods (sample E). The gradual evolution of semiconductor heteronanocrystals toward a type-II optical behavior has been demonstrated for $\mathrm{ZnSe} / \mathrm{CdSe},{ }^{4-5} \mathrm{CdTe} / \mathrm{CdSe},{ }^{6-9}$ and $\mathrm{CdS} /$ $\mathrm{ZnSe}^{17}$ spherical core/shell heteronanocrystals and observed to depend on both the core diameter and the shell thickness. This is consistent with the fact that the position of the energy levels of semiconductor nanostructures is strongly size-dependent, ${ }^{1,2}$ making the relative energy offsets and the degree of carrier localization in heteronanocrystals strongly sensitive to the dimensions (and shape) of both the core and the shell. ${ }^{9,17,35}$ Recent theoretical modeling of $\mathrm{ZnSe} / \mathrm{CdSe}^{35}$ and $\mathrm{CdS} / \mathrm{ZnSe}^{17}$ core/shell nanocrystals has shown that the carrier localization regime becomes dependent on the core diameter and shell thickness in the case of small core diameters, evolving from type-I to type-II through an intermediate regime ("quasi-typeII"), in which one carrier is still delocalized over the entire volume of the heteronanocrystal. Calculations based on the effective mass approximation have also been carried out for a $\mathrm{CdTe} / \mathrm{CdSe}$ core/shell system ${ }^{9}$ (core diameter: $3.9 \mathrm{~nm}$ ) and show that the hole wave function is mostly localized in the core already for rather thin CdSe shells $(0.2 \mathrm{~nm})$, while the electron wave function remains delocalized through the whole core/shell QD up to a shell thickness of $0.9 \mathrm{~nm}$, when it starts to localize

(32) Wuister, S. F.; de Mello Donegá, C.; Meijerink, A. J. Chem. Phys. 2004, $121,4310-4315$

(33) Shabaev, A.; Efros Al, L. Nano Lett. 2004, 4, 1821-1825.

(34) Li, L.; Hu, J.; Yang, W.; Alivisatos, A. P. Nano Lett. 2001, 1, 349-351.

(35) Piryatinski, A.; Ivanov, S. A.; Tretiak, S.; Klimov, V. I. Nano Lett. 2007, 7, $108-115$. in the shell. The transition from type-I to type-II behavior is thus not abrupt, leading to a gradual change in the optical properties as the localization of the electron and hole in different parts of the heteronanostructure becomes more pronounced, in good qualitative agreement with the results reported here. The use of the theoretical models above $e^{9,17,35}$ to quantitatively analyze our results is precluded by the fact that the heteronanocrystals are modeled in refs 9,17 , and 35 as spherically symmetric core/shell nanostructures.

Low PL QYs have been seen as an intrinsic limitation of type-II heteronanostructures, since the slower radiative recombination of indirect excitons should facilitate the dominance of nonradiative recombination. ${ }^{6,12}$ In this context the high PL quantum yields of the $\mathrm{CdTe} / \mathrm{CdSe}$ heteronanocrystals investigated here (as high as $82 \%$ for prolate nanocrystals and $45 \%$ for multipods dispersed in chloroform, see Figure 2) are extremely remarkable, since they are in striking contrast with the extremely low PL QYs reported in the literature for CdTe/ CdSe nanostructures, viz. $\leq 10 \%$ for core/shell $\mathrm{QDs}^{6,8,16}$ and $\sim 0 \%$ for tetrapods, ${ }^{10}$ heteronanorods, ${ }^{11}$ and dumbbarbells. ${ }^{12}$ These high PL QYs indicate relatively slow nonradiative recombination rates, implying low defect concentrations and therefore attesting the high-quality of the CdTe/CdSe heteronanocrystals prepared in this work. The low defect concentration is probably due to the combination of slow heteroepitaxial growth and conditions that favor effective surface passivation, relaxation, and reconstruction. ${ }^{18,19}$ An additional effect of the anisotropic growth is the smaller strain at the $\mathrm{CdTe}-\mathrm{CdSe}$ heterointerfaces compared to spherical core/shell heteronanocrystals. The lattice mismatch between $\mathrm{CdTe}$ and $\mathrm{CdSe}(\sim 6 \%)$ will lead to defects in thick shell spherical nanocrystals, but anisotropic growth will minimize the stress in the heterostructured nanocrystal and reduce the formation of nonradiative traps, since anisotropic heteronanocrystals, such as heteronanorods, can accommodate much larger lattice mismatches than concentric heterostructures. ${ }^{10}$ These results show that type-II heteronanocrystals can have high QYs, despite the longer radiative lifetimes of indirect excitons, provided the concentration of surface and interfacial defects is minimized.

3.3. Temperature Dependence of the Optical Properties. Figure 4 presents the emission spectra of CdTe/CdSe heteronanocrystals (Figure 4a) and $2.6 \mathrm{~nm} \mathrm{CdTe} \mathrm{QDs} \mathrm{(Figure} \mathrm{4b)} \mathrm{at}$ several temperatures $(293-383 \mathrm{~K})$ and compares the temperature-dependent photoluminescence quantum yields of CdTe QDs and a number of CdTe/CdSe heteronanocrystal samples dispersed in decalin (Figure 4c). It can be seen that the PL intensity of CdTe QDs is strongly quenched as the temperature increases, while that of the $\mathrm{CdTe} / \mathrm{CdSe}$ heteronanocrystals remains essentially unaffected (or even shows a modest increase in some cases). The different behavior of CdTe QDs and CdTe/ $\mathrm{CdSe}$ heteronanocrystals regarding thermally activated nonradiative relaxation is also reflected in the PL decay curves (Figure $5)$, which become faster and more nonexponential with increasing temperature for CdTe QDs (Figure 5a), consistent with an increasing contribution of nonradiative recombination to the exciton relaxation. In contrast, the PL decay curves of CdTe/ $\mathrm{CdSe}$ heteronanocrystals (see Figure $5 \mathrm{~b}$ for a representative example) remain (nearly)single-exponential and become slightly slower with increasing temperature, which correlates well with the absence of thermal quenching and the modest increase in 

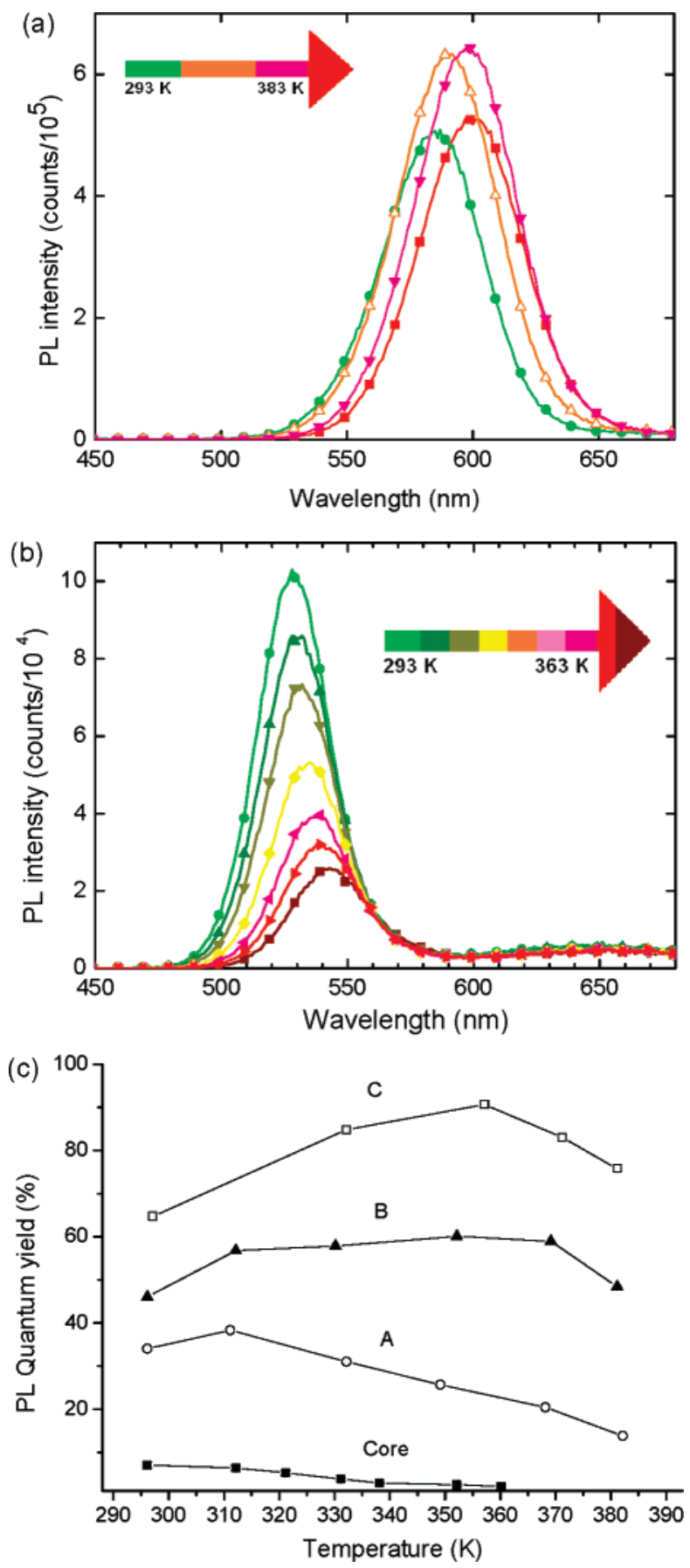

Figure 4. Photoluminescence spectra of (a) CdTe/CdSe heteronanocrystals (Sample B, Table 1) at temperatures between 293 and $383 \mathrm{~K}$ and (b) 2.6 $\mathrm{nm}$ CdTe QDs at temperatures between 293 and 363 K. (c) PL QY in decalin as a function of temperature for $2.6 \mathrm{~nm} \mathrm{CdTe}$ QDs and prolate $\mathrm{CdTe} / \mathrm{CdSe}$ samples (core and samples A, B, and C as in Table 1).

the luminescence QYs observed upon increasing the temperature from 293 to $373 \mathrm{~K}$ (Figure 4). Temperature quenching of the photoluminescence of QDs (e.g., CdSe/ZnS core/shell) is a commonly observed phenomenon, both in colloidal suspensions and in solvent-free systems, such as QDs embedded in polymeric matrices and QD solids, and is ascribed to thermally activated carrier trapping and nonradiative recombination at defects. ${ }^{36-39}$ The behavior of the $\mathrm{CdTe} / \mathrm{CdSe}$ heteronanocrystals is thus quite

(36) Valerini, D.; Creti, A.; Lomascolo, M.; Manna, L.; Cingolani. R.; Anni, M. Phys. Rev. B 2005, 71, 235409/1-6.
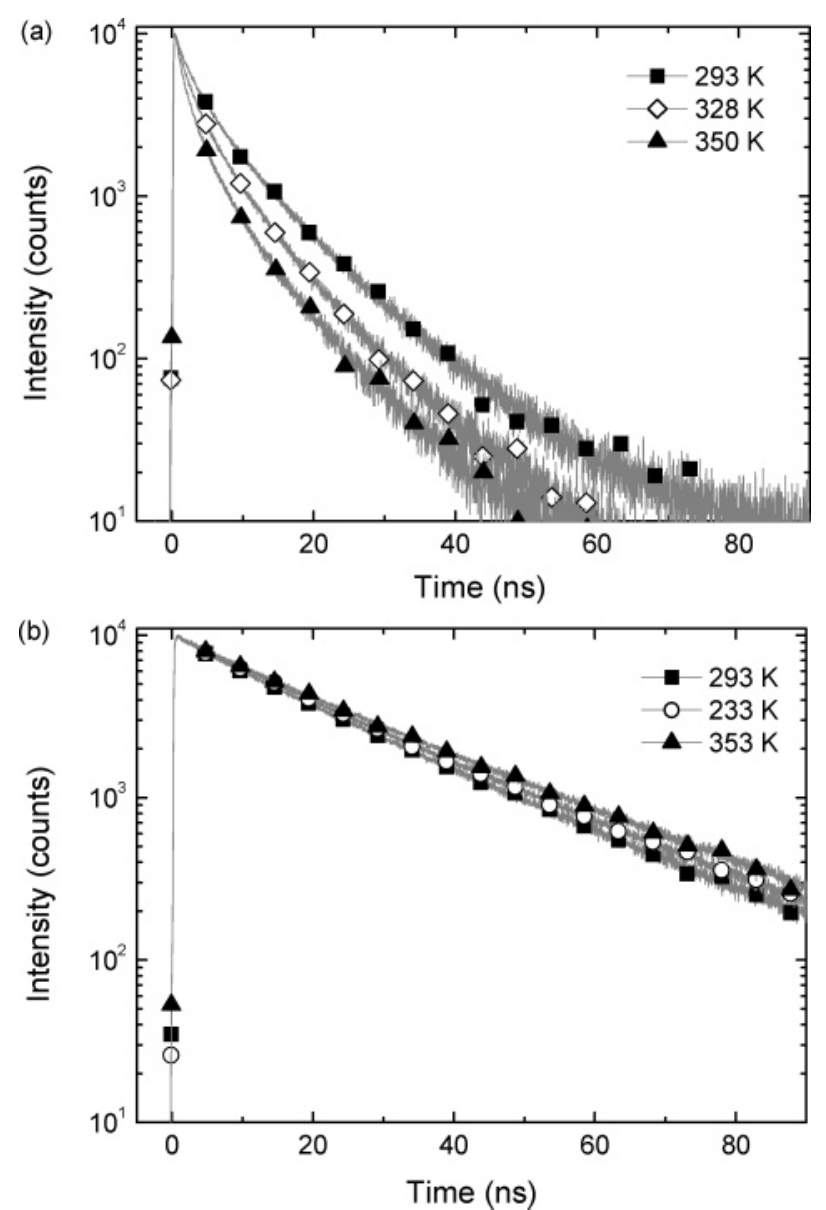

Figure 5. Photoluminescence decay of (a) CdTe QDs and (b) CdTe/CdSe QD (sample C) at different temperatures.

remarkable and unusual, indicating that the defect concentration in these nanocrystals is very low. The modest increase in the PL intensities accompanied by slightly longer exciton lifetimes suggests that the nonradiative recombination rates even decrease a little with increasing temperature. This behavior is reminiscent of the "luminescence temperature antiquenching" effect observed for CdSe QDs capped by linear alkylamines ${ }^{40}$ and is probably also due to a reversible surfactant-assisted surface relaxation (and/or reconstruction).

Figure 4 shows also that the PL peak red shifts as the temperature increases, for both the $\mathrm{CdTe}$ cores and $\mathrm{CdTe} / \mathrm{CdSe}$ heteronanocrystals. The data presented in Figure 6 reveal that this thermally induced PL red shift is larger for the CdTe cores and decreases as the dimension of the CdSe part of the heteronanocrystal increases. The absorption spectra are also observed to red shift with increasing temperature, but a quantitative analysis is complicated by the fact that the lowest energy absorption peak also contains contributions from the lower energy tail of higher energy transitions (the bands are quite broad with respect to the energy separation between subsequent transitions). Since different absorption transitions

(37) Walker, G. W.; Sunder, V. C.; Rudzinski, C. M.; Wun, A. W.; Bawendi, M. G.; Nocera, D. G. Appl. Phys. Lett. 2003, 83, 3555-3557.

(38) Liu, T.-C.; Huang, Z.-L.; Wang, H.-Q.; Wang, J.-H.; Li, X.-Q.; Zhao, Y.D.; Luo, Q.-M. Anal. Chim. Acta 2006, 559, 120-123.

(39) Schöps, O.; Le Thomas, N.; Woggon, U.; Artemyev, M. V. J. Phys. Chem B 2006, 110, 2074-2079.

(40) Wuister, S. F.; de Mello Donegá, C.; Meijerink, A. J. Am. Chem. Soc. 2004, 126, 10397-10402. 

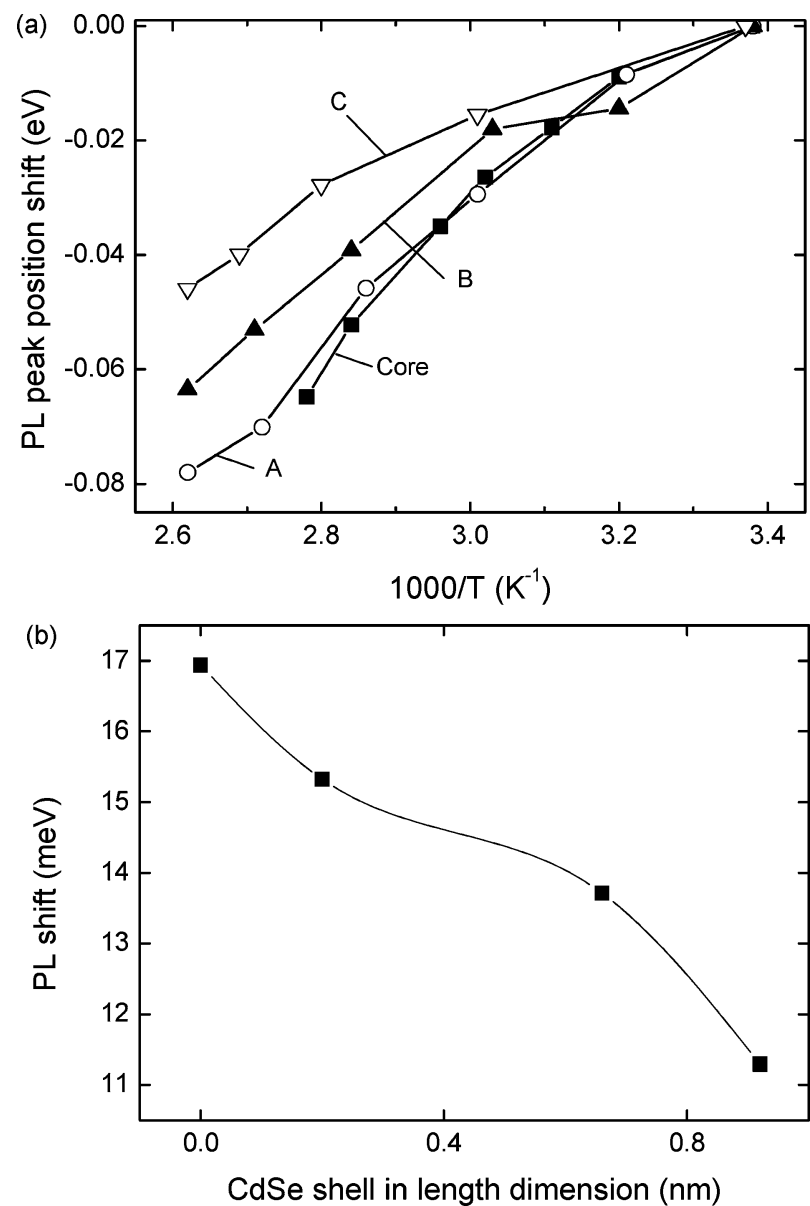

Figure 6. (a) PL peak position as function of temperature for CdTe and $\mathrm{CdTe} / \mathrm{CdSe}$ heteronanocrystals with increasing prolate shell thickness (samples A, B, and C, as defined in Table 1). (b) Total PL emission shift from 293 to $373 \mathrm{~K}$ for $\mathrm{CdTe} / \mathrm{CdSe}$ heteronanocrystals with increasing prolate CdSe shell.

may have different temperature dependences in terms of both energies and oscillator strengths, the maximum of the lowest absorption peak may not reliably reflect the temperature dependence of lowest energy exciton transition. The temperature-dependent shift of the absorption and the emission spectra (as well as the accompanying PL intensity changes discussed above) are fully reversible, indicating that the samples do not undergo any chemical modification and that the temperature dependence of the optical properties can be thus ascribed to a band gap change.

The temperature dependence of the band gap $\left(E_{\mathrm{g}}\right)$ of bulk semiconductors is well described by the Varshni relation, ${ }^{41}$ which has been shown to be also valid for semiconductor nanocrystals: ${ }^{36,42}$

$$
E_{\mathrm{g}}=E_{0}-\alpha \cdot T^{2} /(T+\beta)
$$

where $\alpha$ is the temperature coefficient, $\beta$ is approximately the Debye temperature of the material, and $E_{0}$ is the band gap at 0 $\mathrm{K}$. The values of $\alpha$ and $\beta$ have been obtained for $\mathrm{CdSe} / \mathrm{ZnS}$ nanocrystals $\left[\right.$ viz., $(3.2 \pm 0.2) \times 10^{-4} \mathrm{eV} / \mathrm{K}$ and $220 \pm 30 \mathrm{~K}$, ref 36 and $(4.2 \pm 0.5) \times 10^{-4} \mathrm{eV} / \mathrm{K}$ and $155 \pm 55 \mathrm{~K}$, ref 42 ,

(41) Varshni, Y. P. Physica 1967, 34, 149-154.

(42) Joshi, A.; Narsingi, K. Y.; Manasreh, M. O.; Davis, E. A.; Weaver, B. D. Appl. Phys. Lett. 2006, 89, 131907/1-3.

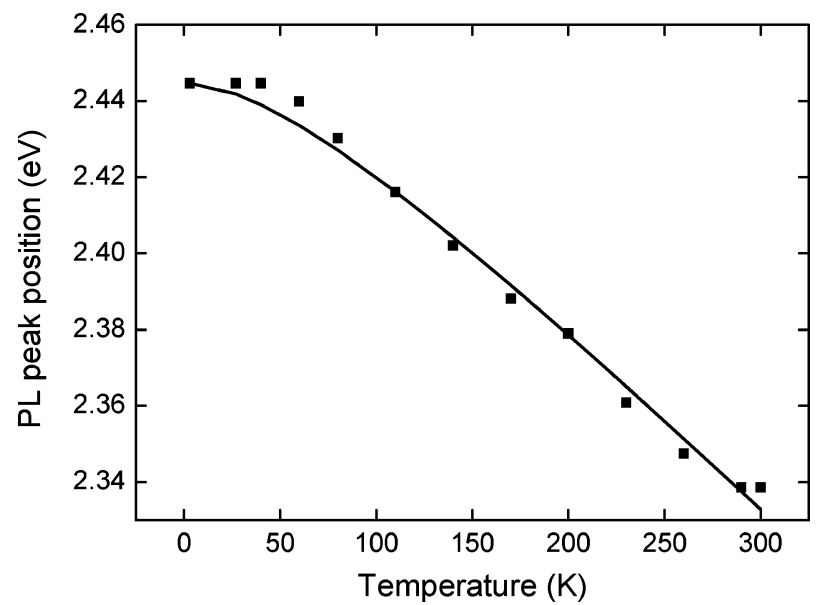

Figure 7. Photoluminescence peak position of $2.6 \mathrm{~nm} \mathrm{CdTe} \mathrm{QDs} \mathrm{as} \mathrm{a}$ function of temperature. The solid line is the best-fit curve with the Varshni relation.

for $\alpha$ and $\beta$, respectively] and are consistent with the values known for bulk CdSe [viz., $(2.8-4.1) \times 10^{-4} \mathrm{eV} / \mathrm{K}$ and $(181-$ $315) \mathrm{K}$ for $\alpha$ and $\beta$, respectively ${ }^{43}$ ]. However, $\alpha$ and $\beta$ values for CdTe nanocrystals are not available in the literature, and the values reported for bulk CdTe are conflicting (e.g., $\alpha=$ $-3 \times 10^{-4} \mathrm{eV} / \mathrm{K}$ in ref 44 and $4.1 \times 10^{-4} \mathrm{eV} / \mathrm{K}$ in ref 45$)$. Therefore, the $\alpha$ and $\beta$ coefficients of CdTe nanocrystals were determined in this work, by fitting the Varshni relation to the temperature dependence of the PL peak positions of $2.6 \mathrm{~nm}$ CdTe QDs in the 3 to $280 \mathrm{~K}$ temperature range (Figure 7 ). The fit yields a band gap of $2.446 \mathrm{eV}$ at $0 \mathrm{~K}, \alpha=(5 \pm 0.47) \times$ $10^{-4} \mathrm{eV} / \mathrm{K}$ and $\beta=102 \pm 32 \mathrm{~K}$, which is close to the Debye temperature $(158 \mathrm{~K})$ reported in literature. ${ }^{43}$ These results imply that the band gap of CdTe depends more strongly on the temperature than that of $\mathrm{CdSe}$, which explains the results reported above (Figure 6). As discussed above (section 3.2), the hole wave function is strongly localized in the CdTe core already for rather thin CdSe shells, while the electron wave function progressively localizes in the CdSe part of the heteronanocrystal as the CdSe dimensions increase. Consequently, the CdSe contribution to the PL energies becomes increasingly larger, leading to a smaller temperature-dependent shift of the PL peak.

\section{Conclusions}

In summary, the preparation methodology developed in this work yields highly efficient anisotropic CdTe/CdSe colloidal heteronanocrystals, with shapes tunable from prolate to branched heteronanocrystals. The high-quality and low defect concentration of these heteronanocrystals is attested by their high photoluminescence quantum yields and negligible thermally induced quenching up to temperatures as high as $373 \mathrm{~K}$, which allowed the observation of reversible thermally induced spectral red shifts. A progressive reduction of the electron-hole wave function overlap and the formation of an indirect exciton are observed as the dimension of the CdSe part of the heteronanoc-

(43) Numerical Data and Functional Relationship in Science and Technology; Hellwege, K. H., Ed.; Landolt-Börnstein, New Series, Group III, Vol. 17, Pt. B; Springer-Verlag: Berlin, 1982.

(44) Camassel, J.; Auvergne, D.; Mathieu, H.; Triboulet, R.; Marfaing, Y. Solid State Commun. 1973, 13, 63-68.

(45) Kosyachenko, L. A.; Sklyarchuk, V. M.; Sklyarchuk, Y. F.; Ulyanitsky, K. S. Semicond. Sci. Technol. 1999, 14, 373-377. 
rystal increases, ultimately leading to a characteristic type-II behavior for the $\mathrm{CdTe} / \mathrm{CdSe}$ multipods. These results show that type-II heteronanostructures can have high photoluminescence quantum yields, which has important consequences for a number of potential applications (e.g., photovoltaic devices, lasers, LEDs).
Acknowledgment. This research has been supported by NanoNed and by the Interreg program OLED+.

Supporting Information Available: X-ray powder diffraction of CdTe and CdTe/CdSe QDs. This material is available free of charge via the Internet at http://pubs.acs.org.

JA0738071 